\title{
Studies for slowly rotating asteroids (168) Sibylla and (346) Hermentaria
}

\author{
Xiaobin Wang ${ }^{1,2}, \dagger$ Karri Muinonen ${ }^{3,4}$, Xianming L. Han $^{5}$ and \\ Chi-Tai Kwok ${ }^{6}$ \\ ${ }^{1}$ Yunnan Observatories, CAS, China \\ P.O. Box 110, Kunming 650011, Yunnan Province, China \\ email: wangxb@ynao.ac.cn \\ ${ }^{2}$ Key laboratory for the structure and evolution of celestial objects, CAS, Kunming 650011, \\ China \\ ${ }^{3}$ Department of Physics, University of Helsinki, \\ Gustaf Hällströmin katu 2a, P.O. Box 64, FI-00014 U. Helsinki, Finland \\ email: karri.muinonen. @helsinki.fi \\ ${ }^{4}$ Finnish Geospatial Research Institute, \\ Geodeetinrinne 2, FI-02430 Masala, Finland \\ ${ }^{5}$ Department of Physics and Astronomy, Butler University \\ Indianapolis, IN, United States \\ email: xhan@butler.edu \\ ${ }^{6}$ Ho Koon Nature Education Cum Astronomical Centre, Sik Sik Yuen, Hong Kong, China
}

\begin{abstract}
Studies for spin parameters and shapes of asteroids provide us with important information about the interior structure of asteroids and the physical processes they have undergone. A large sample of basic physical parameters can help us also understand the evolution of asteroids. There is scarce information for slowly-rotating larger asteroids because more effort is required for observing them. Because of this, we have established an international collaboration to study slowly-rotating asteroids. As the first step of this project, we have observed asteroids (168) Sibylla and (346) Hermentaria in 2014 and 2015 using several telescopes located in China, Chile, and U.S.A. Combining previous photometric data with our new data, we have performed preliminary analyses and obtained spin parameters and shapes with their uncertainties for these two slowly-rotating asteroids for the first time, using the convex inversion method and the virtual photometry Monte Carlo method. A pair of pole solutions for (168) Sibylla are found around $\left(4.3^{\circ}, 53.5^{\circ}\right)$ and $\left(183.5^{\circ}, 52.6^{\circ}\right)$ with a period of $47.0000 \mathrm{~h}$. We have found that the shape of Sibylla resembles an oblate spheroid. For (346) Hermentaria, we have also found a pair of pole solutions around $\left(134.5^{\circ}, 16.7^{\circ}\right)$ and $\left(321.5^{\circ}, 14.5^{\circ}\right)$ with comparable rms-values with a spin period of about $17.79000 \mathrm{~h}$, and a shape resembling a prolate spheroid.
\end{abstract}

Keywords. Minor planets, asteroids, solar system: general, radiative transfer, scattering, methods: data analysis, methods: numerical, methods: statistical, techniques: photometric

\section{Introduction}

More than 690,000 asteroids have been discovered, but only a tiny fraction of them have been studied sufficiently to characterize their basic physical properties (or parameters), such as shape, pole, and spin period. A large sample of such parameters has been used to understand the origin and evolution of the Solar System and the asteroids themselves. In the last decade, knowledge on asteroid shapes and spins has accumulated rapidly. Based

$\dagger$ Present address: Yunnan Observatories, Kunming, Yunnan Province, China. 
on the knowledge, interior structures of asteroids and fundamental physical processes that they have experienced have been inferred at an increasing rate.

For example, the Maxwellian distribution of spin rates for the large asteroids with diameters larger than $40 \mathrm{~km}$ reflects a collision-dominated evolution, whereas the nonMaxwellian distribution for the smaller asteroids in the main belt is due to the combination of the YORP effect and the collisions (Marzari et al. (2011), Pravec \& Harris (2000), and Rubincam (2000)). The cut-off value of the spin rate at $f \sim 10$ revolutions per day (rev/d) implies that most of the small asteroids probably have 'rubble pile' structures. The super-fast rotators are probably monolithic bodies. Some special subsets of asteroids, such as the slow rotators, which probably represent a different physical process for asteroids (Harris (2002)), can open other ways for understanding asteroids, if we learn about their basic physical parameters. Fedorets \& Granvik (2015) and Marciniak et al. (2015) have recently published the first results from similar projects that aim to better constrain the spin and shape properties of slowly-rotating asteroids.

In order to determine the parameters of some selected slowly-rotating asteroids, especially those targets which rotate at rates about $1 \mathrm{rev} / \mathrm{d}$ or $0.5 \mathrm{rev} / \mathrm{d}$, we have established an international collaboration in 2014. In this collaboration, the coordinated observations for the slowly-rotating asteroids are going to be carried out with telescopes distributed at different geographic longitudes. At present, two telescopes located in China, one telescope located at Kitt Peak, Arizona, U.S.A., and one telescope at Cerro Tololo, Chile are available for photometric observations.

Asteroid (168) Sibylla, a C-type asteroid with a diameter of $148 \mathrm{~km}$, have been earlier observed by DiMartino et al. (1994), Wang \& Gu (2004), and Pilcher et al. (2008). The first two groups reported a spin period of $23.82 \mathrm{~h}$ and $24.41 \mathrm{~h}$, respectively, while the third group reported a period of $47.009 \mathrm{~h}$. Asteroid (346) Hermentaria is an S-type main-belt asteroid with a diameter of $111 \mathrm{~km}$. Several groups have made photometric observations of Hermentaria and reported diverging values for its spin period. Harris \& Young (1989) and Bembrick et al. (2004) have reported a spin period of $28.33 \mathrm{~h}$, whereas Wang \& Shi (2002) and Robinson (2011) have reported a spin period of $19.4 \mathrm{~h}$ and $9.7 \mathrm{~h}$, respectively. No pole and shape information about the two asteroids has been obtained previously. Therefore, we have selected asteroids (168) Sibylla and (346) Hermentaria as the first two targets for our study.

In Section 2, we introduce the coordinated observation for the two asteroids. In Section 3, we present our preliminary analysis results with the convex inversion method (Kaasalainen \& Torppa 2001, Kaasalainen et al. 2001) and the virtual photometry Monte Carlo method (Wang et al. 2015). Finally, the discussion for the present study is given in Section 4.

\section{Observations and data reduction}

Based on the published results, the spin period of (168) Sibylla may be $23.5 \mathrm{~h}$ or $47.0 \mathrm{~h}$, and the period of (346) Hermentaria may be $9.7 \mathrm{~h}$, or twice or three times larger. In order to converge on the spin periods for these two asteroids and obtain their poles and shapes, we have carried out coordinated photometric observations in the apparitions of 2014 and 2015 with four telescopes located in China, U.S.A., and Chile. In total, 16 lightcurves have been obtained for the two asteroids. We give the observational details in Table 1, where the first column gives the observation date, the second and third columns give the geocentric and heliocentric distances of the asteroid, the following column gives the solar phase angle, and the last three columns give the aperture of the telescope used, the filter, and the observatory code. Actually, the photometric data for (346) Hermentaria 
Table 1. New observations of (168) Sibylla and (346) Hermentaria. See text.

\begin{tabular}{|c|c|c|c|c|c|c|}
\hline $\begin{array}{l}\text { Date } \\
\text { UT }\end{array}$ & $\begin{array}{c}\Delta \\
(\mathrm{au})\end{array}$ & $\begin{array}{c}r \\
(\mathrm{au})\end{array}$ & $\begin{array}{c}\alpha \\
(\operatorname{deg})\end{array}$ & $\begin{array}{l}\text { Teles- } \\
\text { cope }\end{array}$ & Filter & Obs. code \\
\hline $\begin{array}{l}(168) \\
2015 / 01 / 16.67 \\
2015 / 01 / 17.67 \\
2015 / 01 / 18.67 \\
2015 / 01 / 19.25\end{array}$ & \begin{tabular}{|l}
2.403 \\
2.406 \\
2.409 \\
2.411
\end{tabular} & $\begin{array}{l}3.377 \\
3.377 \\
3.378 \\
3.378\end{array}$ & $\begin{array}{l}2.9 \\
3.1 \\
3.4 \\
3.7\end{array}$ & $\begin{array}{l}0.5 \mathrm{~m} \\
0.5 \mathrm{~m} \\
0.5 \mathrm{~m} \\
0.9 \mathrm{~m}\end{array}$ & $\begin{array}{c}\text { Clear } \\
\text { Clear } \\
\text { Clear } \\
\text { V }\end{array}$ & $\begin{array}{c}\text { Hokoon } \\
\text { Hokoon } \\
\text { Hokoon } \\
695\end{array}$ \\
\hline $\begin{array}{l}(346) \\
2014 / 12 / 11.67 \\
2015 / 01 / 06.25 \\
2015 / 01 / 19.67 \\
2015 / 01 / 20.67 \\
2015 / 01 / 21.67 \\
2015 / 01 / 22.67 \\
2015 / 01 / 23.67 \\
2015 / 01 / 23.25 \\
2015 / 03 / 18.67\end{array}$ & $\begin{array}{l}1.790 \\
1.741 \\
1.791 \\
1.797 \\
1.803 \\
1.809 \\
1.816 \\
1.816 \\
2.438\end{array}$ & $\begin{array}{l}2.697 \\
2.723 \\
2.737 \\
2.738 \\
2.739 \\
2.740 \\
2.741 \\
2.741 \\
2.796\end{array}$ & \begin{tabular}{c|}
10.0 \\
1.6 \\
7.1 \\
7.5 \\
7.9 \\
8.3 \\
8.7 \\
8.6 \\
20.5
\end{tabular} & $\begin{array}{l}1.0 \mathrm{~m} \\
0.9 \mathrm{~m} \\
0.5 \mathrm{~m} \\
0.5 \mathrm{~m} \\
0.5 \mathrm{~m} \\
0.5 \mathrm{~m} \\
0.5 \mathrm{~m} \\
0.6 \mathrm{~m} \\
1.0 \mathrm{~m}\end{array}$ & $\begin{array}{c}\text { Clear } \\
\text { B,V,R } \\
\text { Clear } \\
\text { Clear } \\
\text { Clear } \\
\text { Clear } \\
\text { Clear } \\
\text { R } \\
\text { Clear }\end{array}$ & $\begin{array}{c}286 \\
695 \\
\text { Hokoon } \\
\text { Hokoon } \\
\text { Hokoon } \\
\text { Hokoon } \\
\text { Hokoon } \\
\quad 807 \\
286\end{array}$ \\
\hline
\end{tabular}

Notes: Hokoon represents the Ho Koon Nature Education Cum Astronomical Centre with a geographic position $\left(114.1078^{\circ}, 22.3838^{\circ}\right)$.

from Jan. 5, 2015 are not used due to the influence of lunar light, and the data for (168) Sibylla on Jan. 3 and 12, 2015 are also omitted because of problems with the data.

While carrying out the present study, four telescopes were involved: the 1.0-m telescope at Yunnan Observatories with a $2 \mathrm{k} \times 2 \mathrm{k}$ Andor DW436 CCD camera $\left(0^{\prime \prime} .21 /\right.$ pixel $)$, the 0.9-m SARA telescope at KPNO and 0.6-m SARA telescope at CTIO with ARC $2 \mathrm{k} \times 2 \mathrm{k}$ CCD cameras (usually used in the $2 \times 2$ binning mode, giving a resolution of $0^{\prime \prime} .86 /$ pixel and $0^{\prime \prime} .72 /$ pixel, respectively), and the 0.5 -m telescope at Hokoon with a $3 \mathrm{k} \times 3 \mathrm{k}$ CCD camera $\left(0^{\prime \prime} .59 /\right.$ pixel $)$. The new observational data are reduced in a standard fashion using the IRAF package. All of the scientific images are corrected for the bias, dark current, and flat-field effects. Cosmic rays in the images are identified and removed by a proper threshold. The instrumental magnitude of the objects in the images are measured with an optimal aperture using the APPHOT task of IRAF. Furthermore, the red noise in the photometric data are simulated by using the reference stars in the images, and then removed from the photometric data of the asteroids (Wang \& Gu (2010)).

We have also searched the literatures for existing data of the two asteroids. For (168) Sibylla, 29 lightcurves by Pilcher et al. (2008) are downloaded from the MPC database and 4 lightcurves by Wang \& Gu (2004) are used. 10 lightcurves of (346) Hermentaria from the APC database (Lagerkvist et al. (1993)) and 4 lightcurves from Wang \& Shi (2002) are used. The time stamps for all the data involved are corrected for the light time and then converted into Julian Days in the TDB time system. Figures 1 and 2 show example lightcurves of (168) Sibylla and (346) Hermentaria. In the figures, $\alpha$ represents the solar phase angle of the asteroid, and $\theta$ and $\theta_{0}$ denote angles from the line of sight and the line of incidence to the spin axis of the asteroid, respectively.

\section{Results}

Using the convex inversion method (Kaasalainen \& Torppa (2001), Kaasalainen et al. (2001)) and the virtual-photometry Monte Carlo method (Muinonen et al. (2012), Muinonen et al. (2015)), the photometric data of (168) Sibylla and (346) Hermentaria are analyzed. The procedure contains two parts (for the details, see Wang et al. (2015)). In the first part, unknown parameters (the coefficients of the truncated sphericalharmonics series, spin parameters, and the Lambert law coefficient $c$ ) are estimated with 

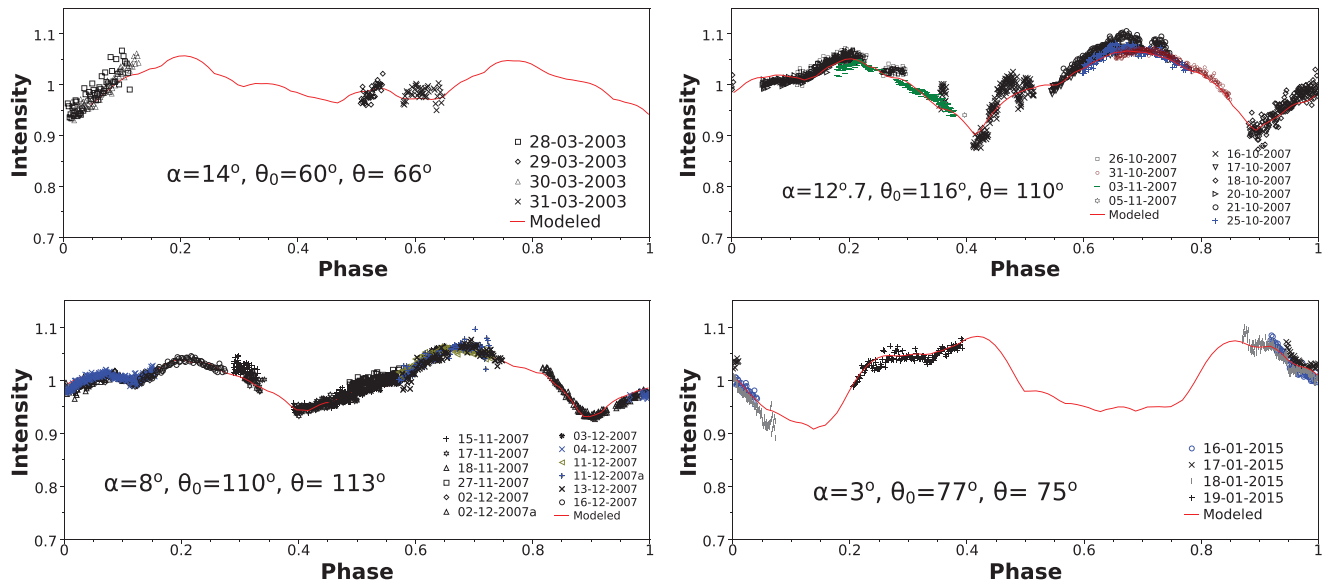

Figure 1. Lightcurves of (168) Sibylla, folded with a period of $47.00 \mathrm{~h}$.
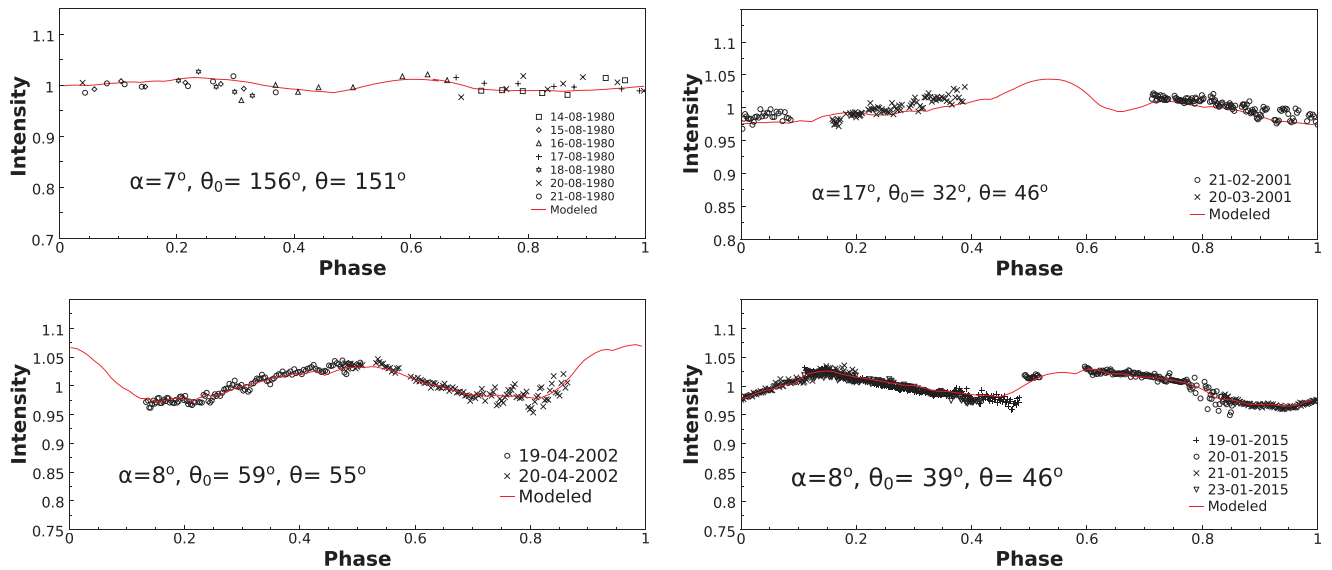

Figure 2. Lightcurves of (346) Hermentaria, folded with a period of $17.79 \mathrm{~h}$.

the convex-inversion method using the Levenberg-Marquardt algorithm. A lower-resolution shape (6 rows in an octant in the triangulation) and lower-order spherical-harmonics series (up to $l=m=6$ ) are adopted when searching for the best period. Then the higher-resolution shape ( 8 rows of triangles as per octant) represented with the higherorder spherical-harmonics series (up to $l=m=8$ ) and the pole are searched with the most significant period as the initial value. In the second part, we carry out virtualphotometry Monte Carlo simulations using the convex inversion for the virtual observations. The parameter distributions composed of the virtual least-squares solutions of the convex inversion are derived. Then, the best values and the uncertainties of the spin parameters are estimated with the help of the statistics of the distributions (here we use the mode and $1 \sigma$-limits of the distributions). The best shape for a given pole solution is the one which is similar to most of the shapes based on a threshold of the Pearson's $\chi^{2}$ quantity (Eq. 1 in Wang et al. (2015)). In what follows, we will describe the results for (168) Sibylla and (346) Hermentaria in detail.

\section{1. (168) Sibylla}

In all, 37 lightcurves of Sibylla distributed at 4 apparitions with the solar phase angle varying from $1.0^{\circ}$ to $14.3^{\circ}$ are used in the present analysis. Based on the previously 

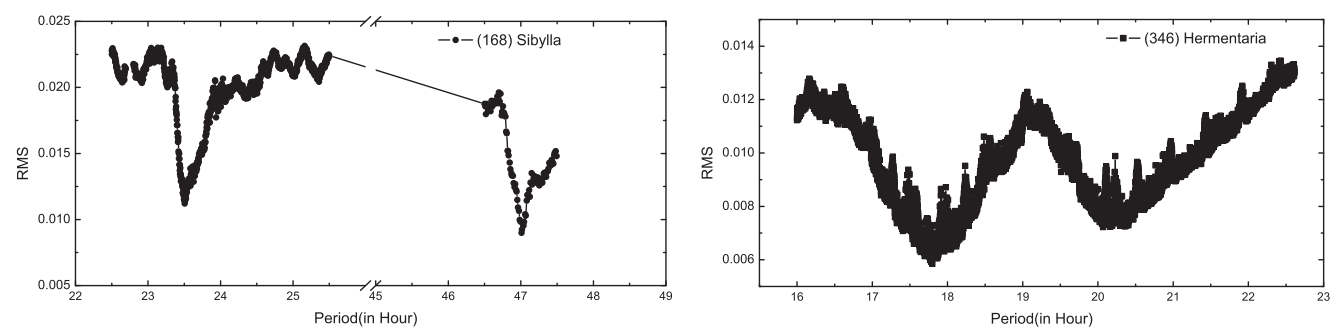

Figure 3. The rms-values of the fit vs. the spin period for (168) Sibylla (left) and (346) Hermentaria (right).
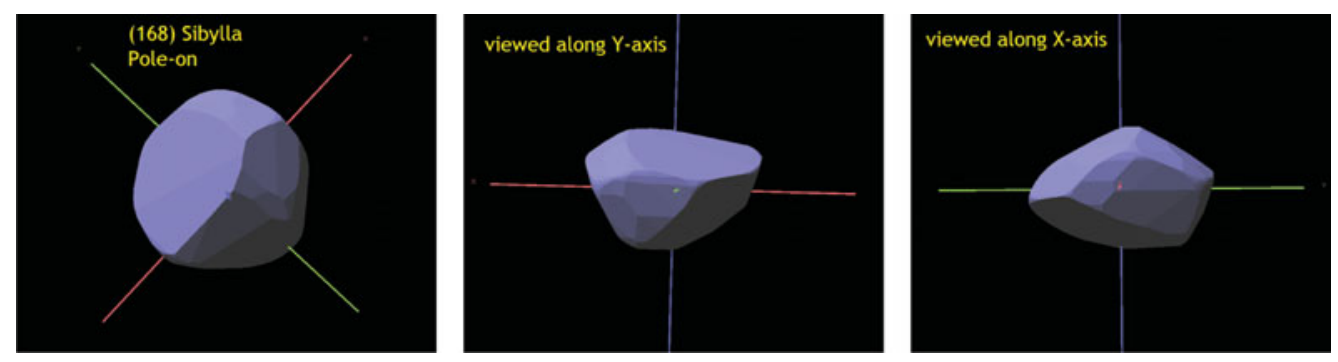

Figure 4. Convex shape of (168) Sibylla.

published results, we let the program search for the spin period at two intervals, $22 \mathrm{~h}$ to $25 \mathrm{~h}$ and $46 \mathrm{~h}$ to $48 \mathrm{~h}$. The left panel of Fig. 3 shows the rms-value of the fit vs. the period distribution for (168) Sibylla. The most significant minimum is located at a period of $47.00 \mathrm{~h}$, whereas the rms-value corresponding to the period of $23.5 \mathrm{~h}$ is $20 \%$ larger than that for $47.00 \mathrm{~h}$. Therefore, we set an initial value of period to $47.00 \mathrm{~h}$ when searching for the pole solution and finding the higher-resolution shape of (168) Sibylla. Using the convex inversion method, we have found two candidate poles around $\left(4.3^{\circ}, 53.5^{\circ}\right)$ and $\left(183.5^{\circ}, 52.6^{\circ}\right)$ (in the ecliptic coordinate of the J2000.0 system) with comparable rmsvalues. Applying the virtual-photometry Monte Carlo method, we have obtained the best spin parameters (see Table 2) and the best shape of (168) Sibylla (see Fig. 4). Figure 5 shows the distributions of spin parameters and the coefficient $c$ of (168) Sibylla. The coefficients of the Lambert law are 0.11 and 0.16 for the two pole solutions.

\section{2. (346) Hermentaria}

We use 23 lightcurves from 4 apparitions when searching for the spin pole and shape of (346) Hermentaria. The solar phase angle of these lightcurves varies from to $2.4^{\circ}$ to $20.5^{\circ}$. The period interval between $8 \mathrm{~h}$ and $29 \mathrm{~h}$ is scanned. The most significant minimum in terms of the rms-value of the fit is located at $17.79 \mathrm{~h}$ (the panel on the right in Fig. 3). Then, its spin parameters and higher-resolution shape are computed with the convexinversion method, and a pair of poles is found around $\left(134.5^{\circ}, 16.7^{\circ}\right)$ and $\left(321.5^{\circ}, 14.5^{\circ}\right)$. The shapes corresponding to the two poles are close to a prolate spheroid (see Fig. 6). With the virtual-photometry Monte Carlo procedure, the best values of the spin parameters with uncertainties (listed in Table 2) are estimated from the distributions in Fig. 7. The best values of the coefficient $c$ are 0.05 and 0.01 for the two poles, which means a smaller weight for the Lambert law in the reflection coefficient for the surface of (346) Hermentaria. 

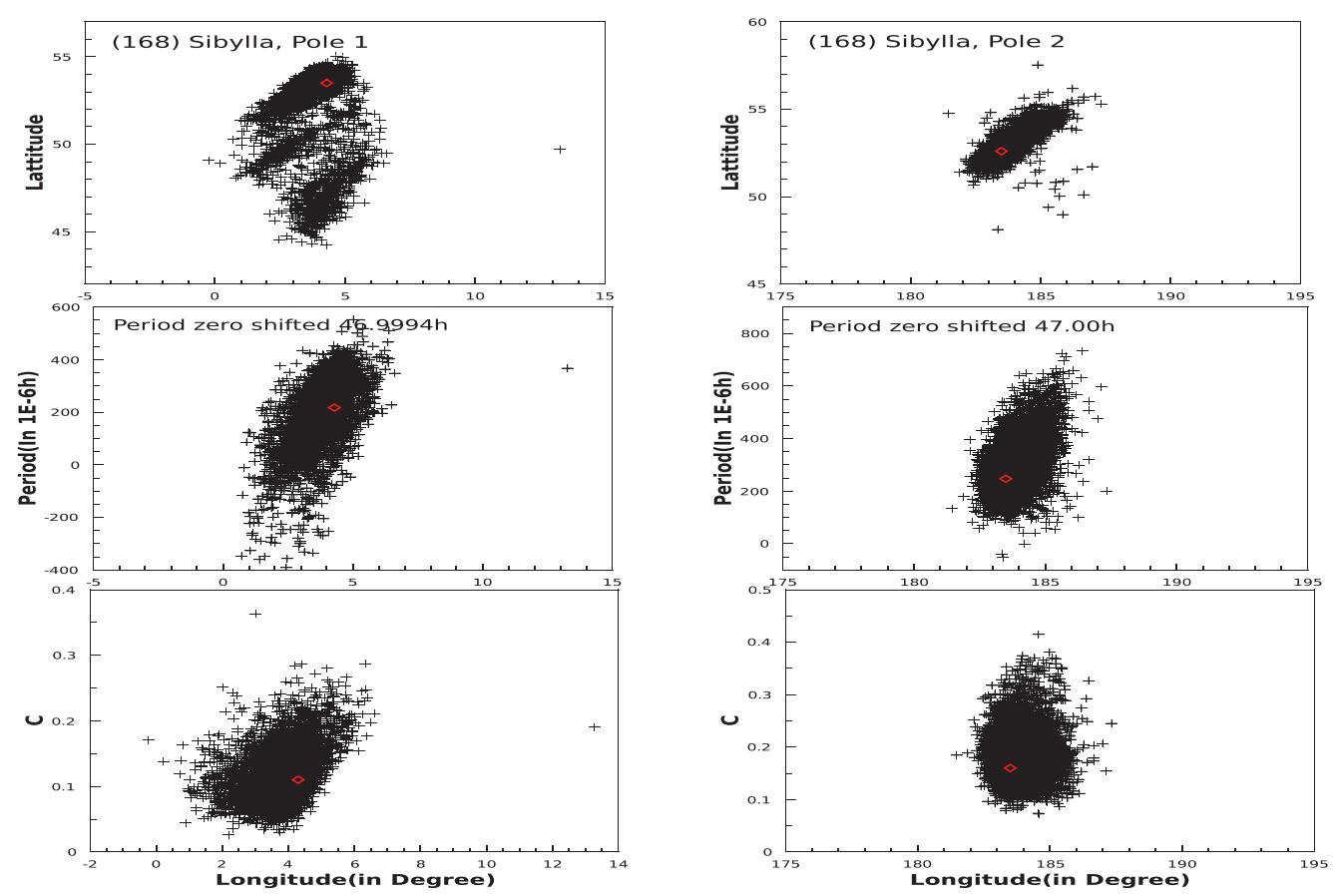

Figure 5. Distributions of spin parameters and $c$-coefficients for (168) Sibylla. The large empty diamond is the Mode value.
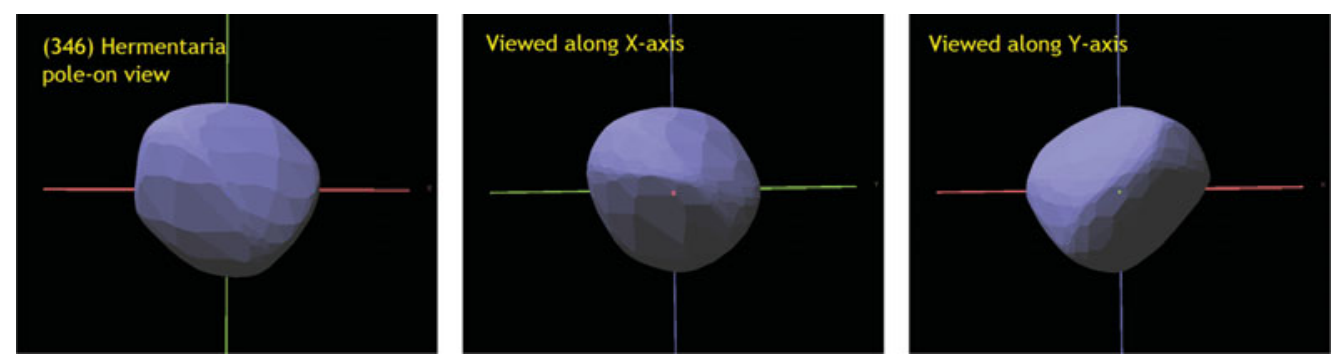

Figure 6. Convex shape of (346) Hermentaria.

\section{Discussion}

We have obtained the spin parameters and shapes for two slowly-rotating asteroids (168) Sibylla and (346) Hermentaria for the first time, using new data from our coordinated observations as well as existing data. We have found two possible pole solutions for (168) Sibylla at around $\left(4.3^{\circ}, 53.5^{\circ}\right)$ and $\left(183^{\circ} .5,52^{\circ} .6\right)$. The period values corresponding to the two poles are $46.9996 \mathrm{~h}$ and $47.0003 \mathrm{~h}$ with an uncertainty of $2.5 \times 10^{-4} \mathrm{~h}$, which are close to the period by Pilcher et al. (2008). The corresponding convex shape of (168) Sibylla is approximately an oblate spheroid with approximate relative axial ratios $a / b=1.0$ and $b / c=1.4$. For the second target (346) Hermentaria, we have also found two pole solutions located at $\left(134^{\circ} .5,16^{\circ} .7\right)$ and $\left(321^{\circ} .5,14^{\circ} .5\right)$ with period values of $17.789993 \mathrm{~h}$ and $17.790038 \mathrm{~h}$, respectively. This period value is different from all of the previous ones. The approximate relative triaxial dimensions (Kaasalainen et al. (2002)) of the derived shape of Hermentaria are $a / b=1.1$ and $b / c=1.0$, that is, the shape is close to a prolate spheroid. 
Table 2. Spin parameters and $c$-coefficients for asteroids (168) Sibylla and (346) Hermentaria.

\begin{tabular}{l|c|c|c|c|}
\hline Asteroid & Period $(\mathrm{h})$ & Long. & Lat. & $\mathrm{c}$ \\
\hline $\begin{array}{l}(168) \\
\text { pole } 1\end{array}$ & $46.999618_{-5.2(-4)}^{+8.9(-5)}$ & $4^{\circ} .3_{-1.1}^{+0.1}$ & $+53^{\circ} .5_{-1.5}^{+0.4}$ & $0.11_{-0.03}^{+0.04}$ \\
pole 2 & $47.000247_{-3.2(-5)}^{+1.4(-4)}$ & $183^{\circ} .5_{-0.3}^{+1.0}$ & $+52^{\circ} .6_{-0.3}^{+1.0}$ & $0.16_{-0.02}^{+0.05}$ \\
\hline \begin{tabular}{l|l|l|l|}
$(346)$ \\
pole 1 \\
pole 2
\end{tabular} & $17.789993_{-2.1(-5)}^{+3.5(-5)}$ & $134^{\circ} .5_{-1.0}^{+1.6}$ & $+16^{\circ} .7_{-1.7}^{+2.3}$ & $0.05_{-0.05}^{+0.07}$ \\
\hline
\end{tabular}

Notes: For example, $+8.9(-5)$ stands for $+8.9 \times 10^{-5}$.
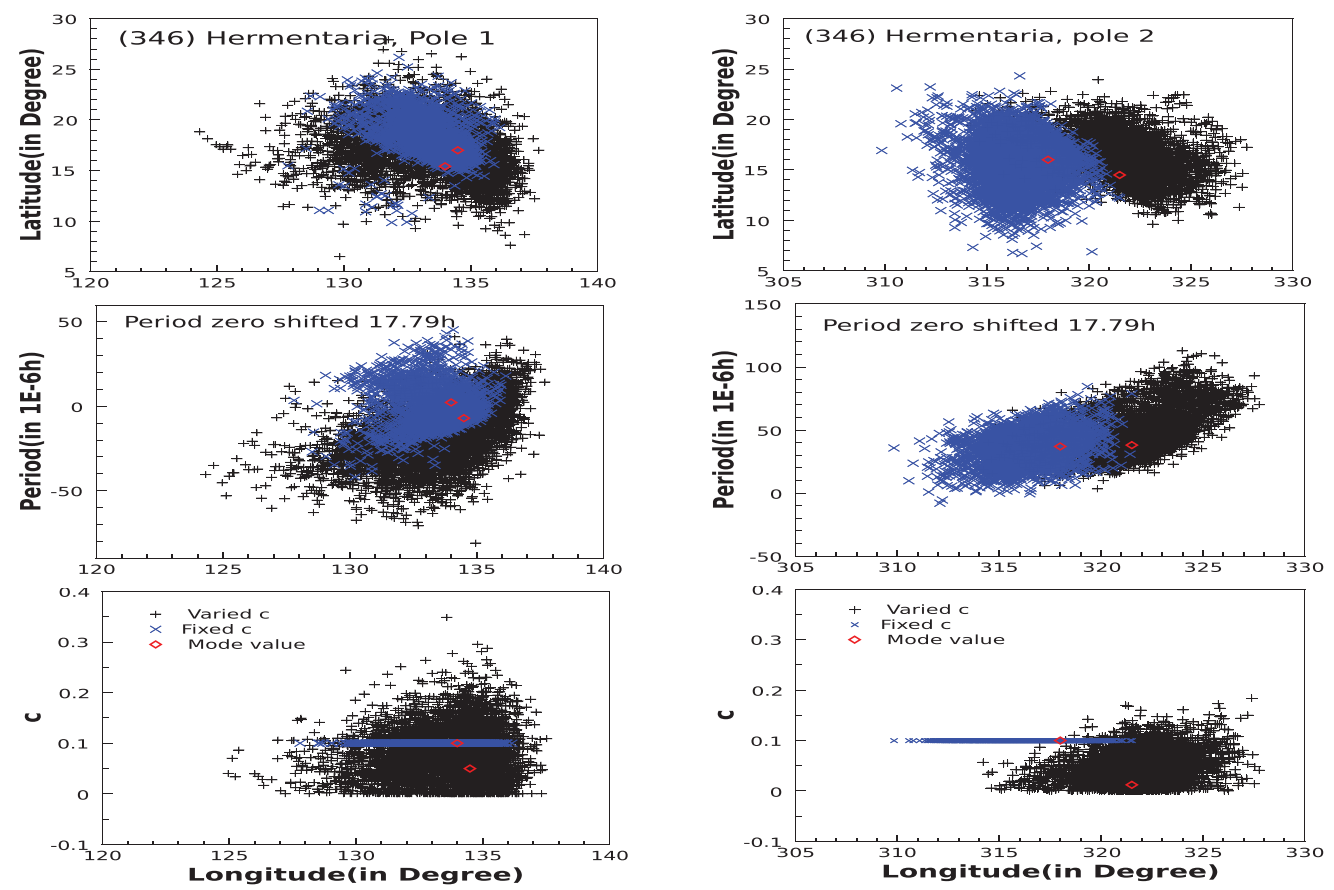

Figure 7. Distributions of spin parameters and $c$-coefficients for (346) Hermentaria. The large empty diamond represents the Mode value.

The uncertainties in the pole solutions for these two asteroids are quite small. This can be due to the fact that the long spin periods allow for highly detailed features to be resolved in the observational lightcurves, which, in turn, can result in highly accurate spin periods. We continue to address the spin periods in our future studies of slowly-rotating asteroids. Overall, more photometric data are needed.

On average, the Lambert coefficient $c$ for (168) Sibylla and (346) Hermentaria are 0.14 and 0.03 , respectively, which implies more weight for the Lambert law in the case of the C-type asteroid Sibylla, in comparison to the S-type asteroid Hermentaria. This result is counter-intuitive as one would expect a larger weight for the higher-albedo Hermentaria, and calls for detailed studies using, for example, the particulate-medium reflection coefficient by Wilkman et al. (2015).

Additionally, virtual-photometry Monte Carlo simulations with a fixed $c=0.1$ have also been carried out for Hermentaria. The distributions of the pole for Hermentaria show slightly shifted centers as compared to the case when the coefficient $c$ is varied. The main 
shift in the case of pole 1 (panel on the left in Fig. 7) is along the latitude direction, whereas the shift for pole 2 is along the longitude direction. We believe it is useful to fit the parameter $c$ even if only relative intensities of asteroids are involved, with which we can derive the spin pole accurately.

\section{Acknowledgements}

The research has been supported by National Natural Sciences Foundations of China (contract No. 11073051) and the Academy of Finland (project No. 1257966).

\section{References}

Bembrick, C. S., Pereghy, B., Ainsworth, A., \& Bolt, G. 2004, MPBu, 31, 52

Di Martino, M., Blanco, C., Riccioli, D., \& De Sanctis, G. 1994, Icarus, 107, 269

Fedorets, G. \& Granvik, M. 2015, Planet. Space Sci., in press

Harris, A. W. \& Young, J. W. 1989, Icarus, 81, 314

Harris, A. W. 2002 Icarus, 156, 184

Kaasalainen, M. \& Torppa, J. 2001, Icarus, 153, 24

Kaasalainen, M., Torppa, J., \& Muinonen, K. 2001, Icarus, 153, 37

Kaasalainen, M., Torppa, J., \& Piironen, J. 2002, Icarus, 159,369

Lagerkvist, C.-I., Magnusson, P., Belskaya, I., Erikson, A., Dahlgren, M., \& Barucci, M. A., Asteroid Photometric Catalog 1993, 3rd Update, Uppsala Astronomical Observatory, Uppsala.

Marciniak, A., Pilcher, F., Oszkiewicz, D., Santana-Ros, T., Urakawac, S., Fauvaud, S., Kankiewicz, P., Tychoniec, L., Fauvaud, M., Hirsch, R., Horbowicz, J., Kaminski, K., Konstanciak, I., Kosturkiewicz, E., Murawiecka, M., Nadolny, J., Nishiyama, K., Okumura, S., Polinska, M., Richard, F., Sakamoto, T., Sobkowiak, K., Stachowski, G., \& Trela, P. 2015, Planet. Space Sci., in press

Marzari, F., Rossi, A., \& Scheeres, D. J. 2011, Icarus, 214, 662

Muinonen, K., Granvik, M., Oszkiewicz, D., Pieniluoma, T., \& Pentikäinen, H. 2012, PESSS, 73, 15.

Muinonen, K., Wilkman, O., Cellino, A., Wang, X., \& Wang, Y. 2015, P\&SS, in press

Robinson, L 2011, http://btboar.tripod.com/lightcurves/

Pilcher, F., Benishek, V., \& Brinsfield, J. W. 2008, MPBu, 35, 104

Pravec, P. \& Harris, A. W. 2000, Icarus, 148,12

Pravec, P., Harris, A. W., Kusnirak, P., Galad, A.,\& Hornoch, K. 2012, Icarus, 221, 365

Rubincam, D. P. 2000, Icarus, 148,2

Wang, X.-B. \& Shi, Y. 2002, EMEP P, 91, 181

Wang, X.-B. \& Gu, S. 2004, EMEPP, 93, 275

Wang, X.-B. \& Gu, S. 2010, EMEPP, 106, 97

Wang, X.-B., Muinonen, K., Wang, Y.-B. et al. 2015, A\& A, 581, A55

Wilkman, O., Muinonen, K., \& Peltoniemi, J. I. 2015, PESSS, in press 